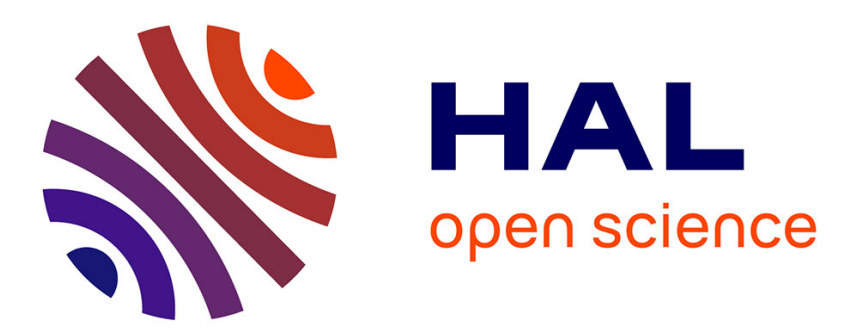

\title{
Explicit homogeneous time-varying stabilizing control of a submarine vehicle (ROV)
}

Adel Khadhraoui, Lotfi Beji, Samir Otmane, Azgal Abichou

\section{To cite this version:}

Adel Khadhraoui, Lotfi Beji, Samir Otmane, Azgal Abichou. Explicit homogeneous time-varying stabilizing control of a submarine vehicle (ROV). 10th International Conference on Informatics in Control, Automation and Robotics (ICINCO 2013), Jul 2013, Reykjavík, Iceland. pp.26-32. hal00879020

\section{HAL Id: hal-00879020 \\ https://hal.science/hal-00879020}

Submitted on 31 Oct 2013

HAL is a multi-disciplinary open access archive for the deposit and dissemination of scientific research documents, whether they are published or not. The documents may come from teaching and research institutions in France or abroad, or from public or private research centers.
L'archive ouverte pluridisciplinaire HAL, est destinée au dépôt et à la diffusion de documents scientifiques de niveau recherche, publiés ou non, émanant des établissements d'enseignement et de recherche français ou étrangers, des laboratoires publics ou privés. 


\title{
Explicit homogeneous time-varying stabilizing control of a submarine vehicle (ROV)
}

\author{
Adel Khadhraoui ${ }^{1}$, Lotfi Beji ${ }^{1}$, Samir Otmane ${ }^{1}$ and Azgal Abichou ${ }^{2}$ \\ ${ }^{1}$ University of Evry, IBISC Laboratory, EA 4526, 40 rue du Pelvoux, 91020 Evry, France \\ 2 Polytechnic School of Tunisia, LIM Laboratory, BP743, 2078 La Marsa, Tunisia \\ adel.khadhraoui@ibisc.univ-evry.fr,Lotfi.Beji@ibisc.univ-evry.fr,Samir.Otmane@ibisc.univ-evry.fr, \\ Azgal.Abichou@ept.rnu.tn
}

Keywords: ROV, kino-dynamic model, time-varying feedback law, stabilization.

Abstract: The stabilizing control problem of a Remotely Operated Vehicle (ROV) for a submarine observing operation is solved explicitly using a homogeneous time-varying controller. We prove that the ROV kino-dynamic model fails the Brockett's necessary condition, consequently, the system's equilibrium cannot be stabilized using continuous pure state feedback laws. Our control strategy is based on a continuous time-varying feedback law taking into account coupling terms due to underactuation and nonlinearities. It is shown that the feedback law stabilizes the ROV at the origin, and this is illustrated by simulations.

\section{INTRODUCTION}

Today, underwater robots are an integral part of scientific equipment to explore the seas and oceans. Many examples have shown that ROVs (Remotely Operating Vehicles) or remotely operated vehicles, and AUVs (Autonomous Underwater Vehicles) are used in many fields and for a variety of applications such as inspection, mapping or bathymetry. However, we can distinguish a depth limit for different types of existing autonomous underwater vehicles. Indeed, from 300 meters, the structure dimensions and characteristics of these vehicles change. One cites AUVs deep as Hugin 3000 sensor of Kongsberg Maritime, the Sea Oracle of Bluefin's Robotics or the Alistar 3000 of ECA, which can reach depths of 3000 meters, and have a high autonomy. An AUV has a significant size and weight and requires significant logistics. However, the ROVs, as of Phantom 500 (Folcher and Rendas, 2001), the ALIVE vehicle of group Cybernetix, the ROV Triton-PR, the AC-ROV (ROV sales) or the underwater vehicle H1000 of the Eca-Hytec, with much less autonomy, they are dedicated for inspection/observation operations in subsea and are not considered for manipulations. From control point of view, which is the key problem to ensure semiautonomy or complete autonomy of the vehicle, the control design becomes a challenge problem due to underactuation (number of inputs less than the number of vehicle's degrees of freedom) and high non- linearities of obtained dynamic models. Robustness of the controller with respect to non stationary subsea environments is also a challenging problem. Underactuation, in the case of an AUV, has been overcome in (Pettersen and Egeland, 1999). The control objective was solved in (Pettersen and Egeland, 1999) by a feedback law that asymptotically stabilizes the origin of AUV using only four, possibly three, control inputs. In (Martins-Encarnacao, 2002), the Lyapunov approach and the backstepping technique were combined and the control achieves the seabed monitoring. The tracking control of an AUV was limited to the horizontal plan in (Lapierre and Soetanto, 2007). A first order sliding mode technique was proposed by Salgado (Folcher and Rendas, 2001) for the Taipan, and supported by experimental results.

Jose et al. (Cunha JP, 1995) presented an adaptive control scheme of dynamic positioning of remotely operated vehicles (ROV) based on a control algorithm called variable structure model-reference adaptive control. In (Santos, 1995), Santos has been studying a law of sensor based control to allow a ROV to monitoring background. In (Nakamura, 2000), the LQI control system and robust control based on $H_{\infty}$ are developed. The stabilizing control problem remains a challenging problem in the case of submarine systems, especially when the vehicle is reduced in sizes and weight. Even if an explicitly time-varying or a discontinuous laws solve the stabilizing control problem for a large underactuated autonomous sys- 
tems (Coron and Rosier, 1994), (Morin and Samson, 1997), (Pettersen and Nijmeijer, 2001), a depth analysis of each vehicle's model is required to design a stabilizing controller. In this paper, we study an ultraportable submarine vehicle, called ROV, and is expected for observation and exploration insubsea historical sites. The ROV is equipped with two cameras and will permits the Tele-exploration in mixed-reality sites. It is procured by the Digital-Ocean 2 project from SUBSEA TECH society. In order to stabilize images, the submarine system should be stabilized for a given desired position and attitude under hydrodynamic effects. Based on the ROV kino-dynamic model, we prove that the Brockett's necessary condition is not satisfied, hence a continuous linear or nonlinear pure state feedback law cannot solve the stabilizing problem. In this paper an explicit homogeneous time-varying control is designed and supported by simulations. The paper is organized as following: In section 2 the dynamics and kinematics of of ROV is described. In section 3 a continuous periodic timevarying feedback law is proposed. The theoretical results are illustrated by simulations in section 4 .

\section{Modelling}

Due to hydrodynamics forces, the ROV model is highly nonlinear and coupled, however, most of nonlinear models are based on simplifying assumptions (Folcher and Rendas, 2001). These assumptions concern often some coupling terms or outright neglect hydrodynamic to calculate or approximate the theory. However, these simplifications, often encountered in aerodynamics study are not recommended in the marine environment (Fossen, 994).

The ROV has a close frame structure (see Fig.1).

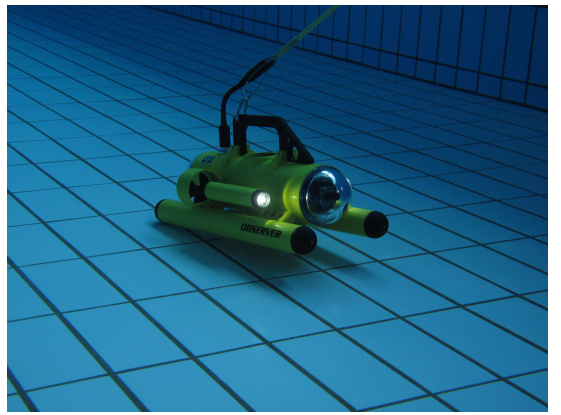

Figure 1: The ROV at $5 \mathrm{~m}$ depth (Chouiten et al., 2012)

This vehicle is actuated with two reversible horizontal thrusters $F_{1 x}$ and $F_{2 x}$ for surge and yaw motion, and a reversible vertical thruster $F_{3 z}$ for heave motion. A 150 meters cable provides electric power to the thrusters and enables communication between the vehicle sensors and the surface equipment (Fig.1).

\subsection{Characteristic of the ROV (SUBSEA TECH)}

\begin{tabular}{|c|c|}
\hline mass $(\mathrm{kg})$ & 10.81 \\
\hline $\begin{array}{c}\text { maximum } \\
\text { speed }\end{array}$ & 3knots $\simeq 1.6(\mathrm{~m} / \mathrm{s})$ \\
\hline dimension $(\mathrm{mm})$ & $L=450, l=270, \mathrm{H}=210$ \\
\hline maximum depth $(\mathrm{m})$ & 150 \\
\hline $\begin{array}{c}\text { coordinate } \\
\text { of } G, \text { w.r.t } R_{v}(\mathrm{~mm})\end{array}$ & $x_{g}=0, y_{g}=0, z_{g}=-16$ \\
\hline $\begin{array}{c}\text { moment of } \\
\text { inertia }\left(\mathrm{kg} / \mathrm{m}^{2}\right)\end{array}$ & $I_{x x}=0.065, I_{y y}=0.216, I_{z z}=0.2$ \\
\hline $\begin{array}{c}\text { product of } \\
\text { inertia }\left(\mathrm{kg} / \mathrm{m}^{2}\right)\end{array}$ & $I_{x y} \simeq I_{y z} \simeq I_{x z} \simeq 10^{-5}$ \\
\hline
\end{tabular}

\subsection{Kinematics}

Two reference frames are considered in the derivation of the ROV kinematic and dynamic equations of motion: The Earth frame $R_{0}$ and the ROV's fixed frame $R_{v}$. The ROV position and the orientation w.r.t $R_{0}$ can

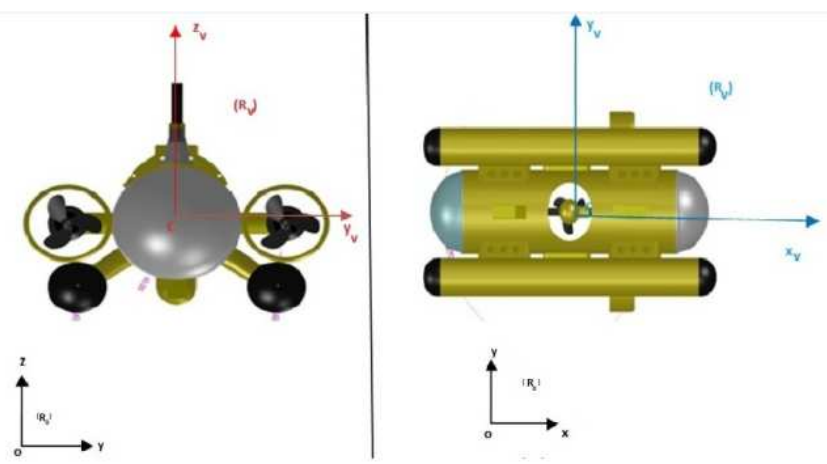

Figure 2: The global and local references frames attached to the ROV.

be respectively described by

$$
\eta_{1}=\left(\begin{array}{c}
x \\
y \\
z
\end{array}\right)_{R_{0}}, \eta_{2}=\left(\begin{array}{c}
\phi \\
\theta \\
\psi
\end{array}\right)_{R_{0}}
$$

with $\phi$ (roll), $\theta$ (pitch) and $\psi$ (yaw) define the attitude and $\eta_{1}=(x, y, z)^{T}$ is the position vector of the ROV. $v_{1}$ is as the linear velocity of the origin and $v_{2}$ is the angular velocity, defined w.r.t $R_{v}$.

$$
\mathrm{v}_{1}=\left(\begin{array}{c}
u \\
v \\
w
\end{array}\right)_{R_{v}}, \mathrm{v}_{2}=\left(\begin{array}{c}
p \\
q \\
r
\end{array}\right)_{R_{v}}
$$


Let us introduce $\eta=\left(\eta_{1}, \eta_{2}\right)^{T} \in \mathbf{R}^{6}$ and $v=$ $\left(v_{1}, v_{2}\right)^{T} \in \mathbf{R}^{6}$. The kinematic of the ROV can be expressed in the following form:

$$
\dot{\eta}_{1}=J_{1}\left(\eta_{2}\right) v_{1}, \dot{\eta}_{2}=J_{2}\left(\eta_{2}\right) v_{2}
$$

The orientation matrices $J_{1}\left(\eta_{2}\right)$ and $J_{2}\left(\eta_{2}\right)$ are as:

$$
\begin{array}{r}
J_{1}\left(\eta_{2}\right)=\left(\begin{array}{ccc}
c \theta c \psi & s \theta s \phi c \psi-s \psi c \phi & s \theta c \phi c \psi+s \psi s \phi \\
c \theta s \psi & s \theta s \phi s \psi+c \psi c \phi & s \theta c \phi s \psi-c \psi s \phi \\
-s \theta & c \theta s \phi & c \theta c \phi
\end{array}\right) \\
J_{2}\left(\eta_{2}\right)=\left(\begin{array}{ccc}
1 & s \phi t \theta & c \phi t \theta \\
0 & c \phi & -s \phi \\
0 & \frac{s \phi}{c \theta} & \frac{c \phi}{c \theta}
\end{array}\right)
\end{array}
$$

It is noticed that the parametrization by the Euler angles have a singularity in $\theta=\frac{\pi}{2}+k \pi$. This parametrization is acceptable because it is impossible for a ROV to reach this singular orientation of 90 degrees pitching angle.

The following shorthand notations for trigonometric functions are used: $c \alpha:=\cos \alpha, s \alpha:=\sin \alpha, t \alpha:=$ $\tan \alpha$ and $T$ is the transpose.

\subsection{Dynamics of the ROV}

According to the CAD software the vehicle has at least two planes of symmetry $x z$ and $y z$. The dynamic model is expressed in the moving frame, centered at the center of gravity.

$$
\begin{gathered}
m\left\{\dot{\mathrm{v}}_{1}+\mathrm{v}_{2} \wedge \mathrm{v}_{1}+\dot{\mathrm{v}}_{2} \wedge r_{g}+\mathrm{v}_{2} \wedge\left(\mathrm{v}_{2} \wedge r_{g}\right)\right\}=\Gamma_{1} \\
I \dot{\mathrm{v}}_{2}+\mathrm{v}_{2} \wedge\left(I \mathrm{v}_{2}\right)+m r_{g} \wedge\left(\dot{\mathrm{v}}_{1}+\mathrm{v}_{2} \wedge \mathrm{v}_{1}\right)=\Gamma_{2}
\end{gathered}
$$

where $\wedge$ represents the cross vector product in $\mathbf{R}^{3}, m$ is the mass of the ROV, $r_{g}=\left[0,0, z_{g}\right]^{T}$ is the postion of the centre of gravity, $I$ is the inertia matrix. $\left[\Gamma_{1}\right.$, $\left.\Gamma_{2}\right]$ is the generalized vector of external forces and moments and the terms $v_{2} \wedge m v_{1}$ and $v_{2} \wedge\left(I v_{2}\right)$ are the centrifugal and Coriolis components. The kinodynamic model of the ROV can be written in the following compact form:

$$
\begin{gathered}
M_{v} \dot{v}+C_{v}(v) \nu+D_{v}(v) \nu+g_{v}\left(\eta_{2}\right)=B_{\tau} \tau \\
\dot{\eta}=J\left(\eta_{2}\right) \nu
\end{gathered}
$$

$M_{v}$ regroups masses and inertia terms (symmetric and definite positive matrix):

$$
M_{v}=\left(\begin{array}{cccccc}
m_{x} & 0 & 0 & 0 & -\alpha_{u q} & 0 \\
0 & m_{y} & 0 & -\alpha_{v p} & 0 & 0 \\
0 & 0 & m_{Z} & 0 & 0 & 0 \\
0 & -\alpha_{v p} & 0 & J_{x} & 0 & 0 \\
-\alpha_{u q} & 0 & 0 & 0 & J_{y} & 0 \\
0 & 0 & 0 & 0 & 0 & J_{z}
\end{array}\right)
$$

where the terms

$$
m_{x}=m-X_{\dot{u}}, m_{y}=m-Y_{\dot{v}}, m_{z}=m-Z_{\dot{w}},
$$

$$
\begin{gathered}
J_{x}=I_{x x}-K_{\dot{p}}, J_{y}=I_{y y}-M_{\dot{q}}, J_{z}=I_{z z}-N_{\dot{r}}, \\
\alpha_{u q}=X_{\dot{q}}-m z_{g}, \alpha_{v p}=m z_{g}+Y_{\dot{p}}
\end{gathered}
$$

$X_{\dot{u}}, Y_{\dot{v}}, Z_{\dot{w}}, K_{\dot{p}}, M_{\dot{q}}, N_{\dot{r}}, X_{\dot{q}}, Y_{\dot{p}}$ are the added masses and inertia, $C_{v}(v)$ is the centrifugal and Coriolis matrix.

$$
C_{v}(v) v=\left(\begin{array}{c}
v_{2} \wedge\left(m+m_{a}\right) v_{1} \\
v_{2} \wedge\left(I_{G}+I_{a}\right) v_{2}+v_{1} \wedge\left(m+m_{a}\right) v_{1}
\end{array}\right) .
$$

with $m_{a}, I_{a}$ are the added masses and inertia, respectively. The hydrodynamic constant matrix $D_{v}$ takes the following form:

$$
D_{v}=-\operatorname{diag}\left\{X_{u}, Y_{v}, Z_{w}, K_{p}, M_{q}, N_{r}\right\} .
$$

The gravitational vector is as:

$$
g_{v}\left(\eta_{2}\right)=\left(\begin{array}{c}
-\left(F_{W}-F_{B}\right) s \theta \\
\left(F_{W}-F_{B}\right) c \theta s \phi \\
\left(F_{W}-F_{B}\right) c \theta c \phi \\
-z_{g} F_{B} c \theta s \phi \\
z_{g} F_{B} s \theta \\
0
\end{array}\right) .
$$

where $F_{B}$ and $F_{W}$ are the buoyancy and gravity magnitudes. The constant matrix $B_{\tau}$ in (17) represents the direction of inputs :

$$
B_{\tau}=\left(\begin{array}{ccc}
1 & 0 & 0 \\
0 & 0 & 0 \\
0 & 0 & 1 \\
0 & 0 & 0 \\
\frac{\alpha_{u q}}{J_{y}} & 0 & 0 \\
0 & 1 & 0
\end{array}\right)
$$

For the following analysis the control inputs are taken as:

$$
\tau=\left(\begin{array}{c}
\tau_{1} \\
\tau_{2} \\
\tau_{3}
\end{array}\right) \triangleq\left(\begin{array}{c}
F_{1 x}+F_{2 x} \\
F_{2 x}-F_{1 x} \\
F_{3 z}
\end{array}\right)
$$

In order to understand the action of different control inputs on the ROV, we need to develop the model given in (3)-(5). Explicitly, the dynamic of the ROV can be written in the form presented below. The six relations related to the dynamic part (4)-(5) are subdivided onto linear and angular accelerations

$$
\begin{aligned}
\dot{u}= & \frac{1}{\delta}\left\{J_{y} X_{u} u+\alpha_{u q} M_{q} q\right. \\
+ & \left(\alpha_{u q} z_{g} F_{B}+J_{y} g\left(F_{W}-F_{B}\right)\right) s \theta+\left(J_{y} m_{y}+\alpha_{u q} \alpha_{v p}\right) v r \\
+ & \left(\alpha_{u q}\left(J_{z}-J_{x}\right)+J_{y} \alpha_{v p}\right) p r-\left(J_{y} m_{z}-\alpha_{u q}^{2}\right) w q \\
- & \left.\alpha_{u q}\left(Z_{\dot{w}}-X_{\dot{u}}\right) w u+\tau_{1}\right\} \\
\dot{v}= & \frac{1}{\delta^{\prime}}\left\{J_{x} Y_{v} v+\alpha_{v p} K_{p} p-\left(J_{x} g\left(F_{W}-F_{B}\right)\right.\right. \\
+ & \left.\alpha_{v p} z_{g} F_{B}\right) c \theta s \phi+\left(\alpha_{v p}^{2}-J_{x} m_{z}\right) w p \\
& -\left(J_{x} m_{x}+\alpha_{v p} \alpha_{u q}\right) u r+\left(J_{x} \alpha_{v p}+\alpha_{v p}\left(J_{y}-J_{z}\right)\right) q r \\
& \left.-\alpha_{v p}\left(\left(Y_{\dot{v}}-Z_{\dot{w}}\right)\right) v w\right\}
\end{aligned}
$$




$$
\begin{aligned}
\dot{w} & =\frac{1}{m_{z}}\left\{Z_{w} w+\left(F_{W}-F_{B}\right) c \theta c \phi+m_{z} u q-m_{y} v p+\alpha_{u q} q^{2}\right. \\
& \left.-\alpha_{v p} p^{2}+\tau_{3}\right\} \\
\dot{p} & =\frac{1}{\delta^{\prime}}\left\{\alpha_{v p} Y_{v} v+m_{y} K_{p} p-\left(\alpha_{v p}\left(F_{W}-F_{B}\right)\right.\right. \\
& \left.-m_{y} z_{g} F_{B}\right) c \theta s \phi+\left(\alpha_{v p} m_{z}-m_{y} \alpha_{v p}\right) w p \\
& -\left(\alpha_{v p} m_{x}+m_{y} \alpha_{u q}\right) u r-\left(\alpha_{v p} \alpha_{u q}+m_{y}\left(J_{y}-J_{z}\right)\right) q r \\
& \left.-m_{y}\left(Y_{\dot{v}}-Z_{\dot{w}}\right) v w\right\} \\
\dot{q} & =\frac{1}{\delta}\left\{\alpha_{u q} X_{u} u+m_{x} M_{q} q+\left(m_{x} z_{g} F_{B}\right.\right. \\
& \left.+\alpha_{u q}\left(F_{W}-F_{B}\right)\right) s \theta+\left(\alpha_{u q} m_{y}+m_{x} \alpha_{v p}\right) v r \\
& +\left(m_{x} \alpha_{u q}-\alpha_{u q} m_{z}\right) w q-\left(\alpha_{u q} \alpha_{v p}-m_{x}\left(J_{z}-J_{x}\right)\right) p r \\
& \left.\left.-m_{x}\left(Z_{\dot{w}}-X_{\dot{u}}\right) w u\right]+\frac{\alpha_{u q}}{J_{y}} \tau_{1}\right\}
\end{aligned}
$$$$
\dot{r}=\frac{1}{J_{z}}\left\{N_{r} r+\left(J_{x}-J_{y}\right) p q-\left(X_{\dot{u}}-Y_{\dot{v}}\right) u v+\left(X_{\dot{q}}+Y_{\dot{p}}\right) u p\right.
$$$$
\left.-\left(X_{\dot{q}}+Y_{\dot{p}}\right) v q+\tau_{2}\right\}
$$

where $\delta=J_{y} m_{x}-\alpha_{u q}^{2}$ and $\delta^{\prime}=J_{y} m_{x}-\alpha_{v p}^{2}$

which are connected to the six kinematic equations,

$$
\begin{aligned}
& \dot{x}=c \theta c \psi u+(s \theta s \phi c \psi-s \psi c \phi) v+(s \theta c \phi c \psi+s \psi s \phi) w \\
& \dot{y}=c \theta s \psi u+(s \theta s \phi s \psi+c \psi c \phi) v+(s \theta c \phi s \psi-c \psi s \phi) w \\
& \dot{z}=-s \theta u+c \theta s \phi v+c \theta c \phi w \\
& \dot{\phi}=p+s \phi t \theta q+c \phi t \theta r \\
& \dot{\theta}=c \phi q-s \phi r \\
& \dot{\psi}=\frac{s \phi}{c \theta} q+\frac{c \phi}{c \theta} r
\end{aligned}
$$

Remark 2.1. There is no direct thrust control to the lateral velocity $v$ and the roll moment $p$. Underactuation can be explained there from.

\section{Stabilizing feedback law}

We will show first, that it is not possible to stabilize the ROV using a feedback law that is continuous and function of the state only. This follows from results given by Brockett (Brockett, 1983), Coron and Rosier (Coron and Rosier, 1994) . The problem is then not solvable using linearization and linear control theory or classical nonlinear control theory like feedback linearization. Thus, we propose a continuous periodic time-varying feedback law that stabilizes the ROV using only three available inputs. The next proposition shows that Brockett's condition cannot meet.

Proposition 3.1. The system (3)-(5) cannot be stabilized by a time invariant smooth pure-state feedback law.
Proof. Let us consider $\varepsilon=\left(\varepsilon_{1}, 0\right)^{T}$, with $\varepsilon_{1} \neq 0$. From equation (7) we get $v=0$, since $J_{2}^{T} J_{2}$ is an invertible matrix. Therefore, equation (17) leads to:

$$
B_{\tau} \tau-g_{v}\left(\eta_{2}\right)=M_{v} \varepsilon_{1}
$$

Then, if we take $\varepsilon_{1}=\left(0,0,0, \varepsilon_{0}, 0,0\right)^{T}$ with $\varepsilon_{0} \neq 0$, we get

$$
\begin{aligned}
& \left(\alpha_{u q} z_{g} F_{B}+J_{y}\left(F_{B}-F_{W}\right)\right) s \theta+\tau_{1}=0 \\
& \left(J_{x}\left(F_{W}-F_{B}\right)-\alpha_{v p} z_{g} F_{B}\right) c \theta s \phi=0 \\
& \left(F_{W}-F_{B}\right) c \theta c \phi+\tau_{3}=0 \\
& \left(\alpha_{v p}\left(F_{W}-F_{B}\right)-m_{y} z_{g} F_{B}\right) c \theta s \phi=\varepsilon_{0} \\
& \left(m_{x} z_{g} F_{B}+\alpha_{u q}\left(F_{B}-F_{W}\right)\right) s \theta+\frac{\alpha_{u q}}{J_{y}} \tau_{1}=0
\end{aligned}
$$

$\tau_{2}=0$

As $\left(J_{x}\left(F_{W}-F_{B}\right)-\alpha_{v p} z_{g} F_{B}\right) \neq 0$, We can deduce from the second equation that $\theta=k \pi+\frac{\pi}{2}$ or $\phi=k \pi$. Further, the fourth equation implies that $\varepsilon_{0}=0$ which is impossible.

As a result, from (Brockett, 1983), we cannot stabilize the ROV by a continuous pure-state feedback. However Coron's theorem (Coron, 1992) proves that time periodic continuous feedback is sufficient to stabilize the system to a point. The main result is given by the following theorem.

Theorem 3.2. Consider the following functions

$$
\begin{aligned}
q_{d} & =-k^{x} x-k^{u} u-k^{\theta} \theta \\
& +\frac{k^{v} v+k^{y} y+k^{\phi} \phi+k^{p} p}{\sqrt{|v|+|y|+|\phi|+|p|}} \sin (t / \varepsilon) \\
w_{d} & =-k^{z} z \\
r_{d} & =-k^{\psi} \psi+2 \sin (t / \varepsilon) \sqrt{|v|+|y|+|\phi|+|p|}
\end{aligned}
$$

and the time-varying feedback law

$$
\begin{aligned}
\tau_{1} & =\frac{J_{y}}{\alpha_{u q}}\left\{\left(\delta k^{q}-m_{x} M_{q}\right) q-\delta k^{q} q_{d}-m_{x} z_{g} F_{B} \theta\right. \\
& \left.-\alpha_{u q}\left(X_{u} u-\left(F_{W}-F_{B}\right) \theta\right)\right\} \\
\tau_{2} & \left.=\left(J_{z} k^{r}-N_{r}\right) r-J_{z} k^{r} r_{d}\right) \\
\tau_{3} & =\left(m_{z} k^{w}-Z_{w}\right) w-m_{z} k^{w} w_{d}-\left(m_{z} u q+\alpha_{u q} q^{2}\right)
\end{aligned}
$$

for a suitable choice of the positive parameters: $k^{x}, k^{y}, k^{z}, k^{p}, k^{q}, k^{r}, k^{\phi}, k^{\theta}, k^{\psi}, k^{u}, k^{v}$ and $k^{w}$ there exists $\varepsilon_{0}$ such that for any $\varepsilon \in\left(0, \varepsilon_{0}\right]$ and large enough $k^{q}, k^{r}$ and $k^{w}$ the feedback (16) and (17) stabilize locally exponentially the kino-dynamic model (14)-(15).

Proof. Let us introduce the following dilation $\Delta_{\lambda}^{r}(\mathrm{v}, \eta, t)=$

$\left(\lambda u, \lambda^{2} v, \lambda^{2} w, \lambda^{2} p, \lambda q, \lambda r, \lambda x, \lambda^{2} y, \lambda^{2} z, \lambda^{2} \phi, \lambda \theta, \lambda \psi\right)$

The linearized system from (14)-(15) can be rewritten as $(c \phi=c \theta=c \psi=1, s \phi \simeq \phi, s \theta \simeq \theta, s \psi \simeq \psi)$

$$
\left(\begin{array}{c}
\dot{\mathrm{v}} \\
\dot{\eta}
\end{array}\right)=f(\mathrm{v}, \eta, t)+g(\mathrm{v}, \eta, t)
$$


with

$$
f(\nu, \eta, t)=\left(\begin{array}{c}
\frac{1}{\delta}\left\{J_{y} X_{u} u+\alpha_{u q} M_{q} q+\left(\alpha_{u q} z_{g} F_{B}\right.\right. \\
\left.-J_{y}\left(F_{W}-F_{B}\right) \theta+\tau_{1}\right\} \\
\frac{1}{\delta^{\prime}}\left\{J_{x} Y_{v} v+\alpha_{v p} K_{p} p+\left(J_{x} g(m-\rho \nabla)\right.\right. \\
\left.-\alpha_{v p} z_{g} F_{B}\right) \phi-\left(J_{x} m_{x}+\alpha_{v p} \alpha_{u q}\right) u r \\
\left.+\left(J_{x} \alpha_{u q}+\alpha_{v p}\left(J_{y}-J_{z}\right)\right) q r\right\} \\
\frac{1}{m_{z}}\left\{Z_{w} w+\left(F_{W}-F_{B}\right)+m_{z} u q+\alpha_{u q} q^{2}\right. \\
\left.+\tau_{3}\right\} \\
\frac{1}{\delta^{\prime}}\left\{\alpha_{v p} Y_{v} v+m_{y} K_{p} p+\left(\alpha_{v p}\left(F_{W}-F_{B}\right)\right.\right. \\
\left.-m_{y} z_{g} F_{B}\right) \phi-\left(\alpha_{v p} m_{x}+m_{y} \alpha_{u q}\right) u r \\
\left.+\left(\alpha_{v p} \alpha_{u q}+m_{y}\left(J_{y}-J_{z}\right)\right) q r\right\} \\
\frac{1}{\delta}\left\{\alpha_{u q} X_{u} u+m_{x} M_{q} q+\left(m_{x} z_{g} F_{B}\right.\right. \\
\left.\left.-\alpha_{u q}\left(F_{W}-F_{B}\right)\right) \theta+\frac{\alpha_{u q}}{J_{y}} \tau_{1}\right\} \\
\frac{1}{J_{z}}\left\{N_{r} r+\tau_{2}\right\} \\
v \\
w \\
p \\
q \\
r
\end{array}\right.
$$

and $g(v, \eta, t)$ is the remaining terms. It is clear that $f(\mathrm{v}, \eta, t)$ defines a periodic, continuous homogeneous of degree zero with respect to the dilation. Also, the function $g(v, \eta, t)$ is continuous and defines a sum of homogeneous vector field of degree strictly positive with respect to the dilation.

To prove the stability of system (14)-(15) it is sufficient to show that

$$
\left(\begin{array}{c}
\dot{v} \\
\dot{\eta}
\end{array}\right)=f(v, \eta, t)
$$

is locally asymptotically stable (as shown in (Coron, 1998)). To this purpose, let us consider the following reduced system obtained from (20), by tacking $q \triangleq q_{d}, p \triangleq r_{d}$ and $w \triangleq w_{d}$ as new control variables. We have obtained the following resulting system:

$$
\left(\begin{array}{c}
\dot{u} \\
\dot{v} \\
\dot{p} \\
\dot{x} \\
\dot{y} \\
\dot{z} \\
\dot{\phi} \\
\dot{\theta} \\
\dot{\psi}
\end{array}\right)=\left(\begin{array}{c}
\gamma_{q} q_{d}+\gamma_{\theta} \theta \\
\alpha_{1} v+\alpha_{2} \phi+\alpha_{3} q_{d} r_{d}+\alpha_{4} u r_{d}+\alpha_{5} p \\
\beta_{1} v+\beta_{2} \phi+\beta_{3} q_{d} r_{d}+\beta_{4} u r_{d}+\beta_{5} p \\
u \\
v \\
w_{d} \\
p \\
q_{d} \\
r_{d}
\end{array}\right)
$$

where

$$
\begin{gathered}
\alpha_{1}=\frac{J_{x} Y_{v}}{\delta^{\prime}}, \beta_{1}=\frac{1}{\delta^{\prime}} \alpha_{v p} Y_{v}, \\
\alpha_{2}=\frac{1}{\delta^{\prime}}\left(J_{x}\left(F_{W}-F_{B}\right)-\alpha_{v p} z_{g} F_{B}\right), \\
\alpha_{3}=\frac{1}{\delta^{\prime}}\left(J_{x} \alpha_{u q}+\alpha_{v p}\left(J_{y}-J_{z}\right)\right), \\
\alpha_{4}=\frac{-1}{\delta^{\prime}}\left(J_{x} m_{x}+\alpha_{v p} \alpha_{u q}\right),
\end{gathered}
$$

$$
\begin{gathered}
\alpha_{5}=\frac{1}{\delta^{\prime}} \alpha_{v p} K_{p}, \beta_{5}=\frac{1}{\delta^{\prime}} m_{y} K_{p} \\
\beta_{2}=\frac{1}{\delta^{\prime}}\left(\alpha_{v p}\left(F_{W}-F_{B}\right)-m_{y} z_{g} F_{B}\right) \\
\beta_{3}=\frac{1}{\delta^{\prime}}\left(-\alpha_{u q} \alpha_{v p}-m_{y}\left(J_{y}-J_{z}\right)\right) \\
\beta_{4}=-\frac{1}{\delta^{\prime}}\left(\alpha_{v p} m_{x}+\alpha_{u q} m_{y}\right) \\
\gamma_{q}=\frac{M_{q}}{\delta}\left(\alpha_{u q}-\frac{J_{y}}{\alpha_{u q}} m_{x}\right) \\
\gamma_{\theta}=\frac{z_{g} F_{B}}{\delta}\left(\alpha_{u q}-\frac{J_{y}}{\alpha_{u q}} m_{x}\right)
\end{gathered}
$$

The controls $q_{d}, r_{d}$, and $w_{d}$ are given by (16). One verifies that the origin of the closed loop system is asymptotically stable, details are in(Coron, 1998). Indeed, the vector field associated with the right-hand side of the closed loop system is continuous periodic and homogeneous of degree zero with respect to the dilation. Due to the periodic time-variant control, the resulting system is a periodic time-varying system, which can be written in the form,

$$
\left(\begin{array}{c}
\dot{v} \\
\dot{\eta}
\end{array}\right)=h(v, \eta, t / \varepsilon)
$$

We approximate this system by an averaged system which is autonomous (M'Closkey and Murray, 1997). The averaged system is defined as $(\dot{\mathrm{v}}, \dot{\eta})^{T}=h_{0}(\mathrm{v}, \eta)$ where $h_{0}(\mathrm{v}, \eta)=\int_{0}^{T_{t}} h_{0}(\mathrm{v}, \eta, t / \varepsilon) d t\left(T_{t}\right.$ is the period $)$. Now, the corresponding averaged system is given by:

$$
\left(\begin{array}{c}
\dot{u} \\
\dot{v} \\
\dot{p} \\
\dot{x} \\
\dot{y} \\
\dot{z} \\
\dot{\phi} \\
\dot{\theta} \\
\dot{\psi}
\end{array}\right)=\left(\begin{array}{c}
\gamma_{q}\left(-k^{x} x-k^{u} u-k^{\theta} \theta\right)+\gamma_{\theta} \theta \\
\left(\alpha_{1}+\alpha_{3} k^{v}\right) v+\left(\alpha_{2}+\alpha_{3} k^{\phi}\right) \phi \\
+\left(\alpha_{5}+\alpha_{3} k^{p}\right) p-\alpha_{4} k^{\psi} u \psi+\alpha_{3} k^{y} y \\
+\alpha_{3} k^{\psi} \psi\left(k^{x} x+k^{u} u+k^{\theta} \theta\right) \\
\left(\beta_{1}+\beta_{3} k^{v}\right) v+\left(\beta_{2}+\beta_{3} k^{\phi}\right) \phi \\
+\left(\beta_{5}+\beta_{3} k^{p}\right) p-\beta_{4} k^{\psi} u \psi+\beta_{3} k^{y} y \\
+\beta_{3} k^{\psi} \psi\left(k^{x} x+k^{u} u+k^{\theta} \theta\right) \\
u \\
v \\
-k^{z} z \\
p \\
-k^{x} x-k^{u} u-k^{\theta} \theta \\
-k^{\psi} \psi
\end{array}\right)
$$


The linear part of (23) is given by:

$$
\left(\begin{array}{c}
\dot{v} \\
\dot{p} \\
\dot{u} \\
\dot{x} \\
\dot{y} \\
\dot{z} \\
\dot{\phi} \\
\dot{\theta} \\
\dot{\psi}
\end{array}\right)=\left(\begin{array}{c}
\left(\alpha_{1}+\alpha_{3} k^{v}\right) v+\left(\alpha_{2}+\alpha_{3} k^{\phi}\right) \phi \\
+\alpha_{3} k^{y} y+\left(\alpha_{5}+\alpha_{3} k^{p}\right) p \\
\left(\beta_{1}+\beta_{3} k^{v}\right) v+\left(\beta_{2}+\beta_{3} k^{\phi}\right) \phi \\
+\beta_{3} k^{y} y+\left(\beta_{5}+\beta_{3} k^{p}\right) p \\
\gamma_{q}\left(-k^{x} x-k^{u} u-k^{\theta} \theta\right)+\gamma_{\theta} \theta \\
u \\
v \\
-k^{z} z \\
p \\
-k^{x} x-k^{u} u-k^{\theta} \theta \\
-k^{\psi} \psi
\end{array}\right)
$$

Hence, the stability study of the system can be reduced to the following tow sub-systems:

$$
\left(\begin{array}{c}
\dot{u} \\
\dot{x} \\
\dot{\theta}
\end{array}\right)=\left(\begin{array}{c}
\gamma_{q}\left(-k^{x} x-k^{u} u-k^{\theta} \theta\right)+\gamma_{\theta} \theta \\
u \\
-k^{x} x-k^{u} u-k^{\theta} \theta
\end{array}\right)
$$

and

$$
\left(\begin{array}{c}
\dot{v} \\
\dot{p} \\
\dot{y} \\
\dot{\phi}
\end{array}\right)=\left(\begin{array}{c}
\left(\alpha_{1}+\alpha_{3} k^{v}\right) v+\left(\alpha_{2}+\alpha_{3} k^{\phi}\right) \phi \\
+\alpha_{3} k^{y} y+\left(\alpha_{5}+\alpha_{3} k^{p}\right) p \\
\left(\beta_{1}+\beta_{3} k^{v}\right) v+\left(\beta_{2}+\beta_{3} k^{\phi}\right) \phi \\
+\beta_{3} k^{y} y+\left(\beta_{5}+\beta_{3} k^{p}\right) p \\
v \\
p
\end{array}\right)
$$

Now, it is clear that for a suitable gain parameters, the origin of the subsystems (25) and (26) is obviously asymptotically stable. Therefore, the origin of the system (24) is asymptotically stable. As a result, the origin of system (23) is locally asymptotically stable. The exponential stability of the origin of system (21) follows by direct application of corollary 1 (Morin and Samson, 1997).

\section{Simulation Results}

In this section, we give a numerical simulation to illustrate our theoretical results. Before starting, we will present the system parameter values (IS units) used for simulations. The added masses and hydrodynamic coefficients are calculated from the CAD-geometry.

- $m=10.84$ : mass of the ROV.

- $m_{x}=11.8910, m_{y}=11.1948, m_{z}=11.1948$.

- added masses $X_{\dot{u}}=-1.0810, Y_{\dot{v}}=Z_{\dot{w}}=$ $-0.3848, K_{\dot{p}}=0, M_{\dot{q}}=N_{\dot{r}}=-0.0075, X_{\dot{q}}=$ $-1.0885, Y_{\dot{p}}=-0.3848$.
- hydrodynamic coefficients $X_{u}=-2.4674, Y_{v}=$ $-2.4674, Z_{w}=-2.4674, K_{p}=-0.928, M_{q}=$ $-5.3014 \times 10^{-6}, N_{r}=-5.3014 \times 10^{-6}$.

- inertial parameters around the principal axes of inertia $J_{x}=0.065, J_{y}=0.023, J_{z}=0.1995$.

- buoyancy and gravity magnitudes: $F_{B}=10.7, F_{W}=m g$ where $g=9.81$.

- terms $\delta=1.5016, \delta^{\prime}=16.0238$, $\alpha_{u q}=1.2615, \alpha_{v p}=0.2119$.

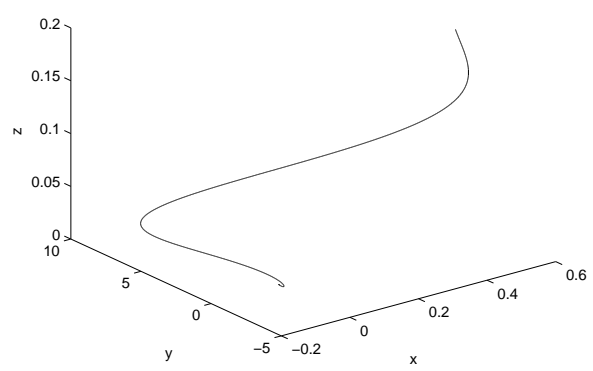

Figure 3: trajectory of the ROV

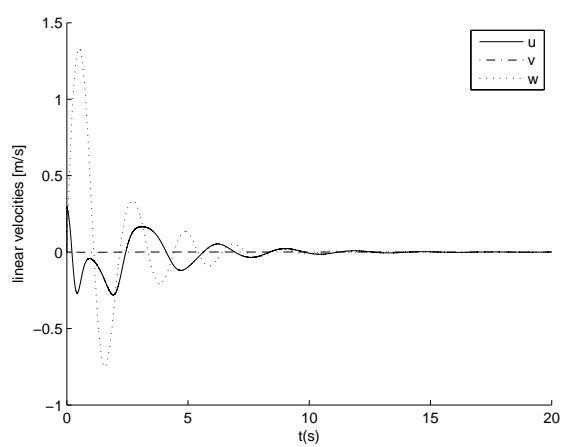

Figure 4: Behaviour of states $u, v$ and $w$

Guided by linear control theory applied to the linearization, we have chosen the following control parameters: $k^{x}=5, k^{y}=10, k^{z}=10$, $k^{p}=10, k^{q}=10, k^{r}=2.5, k^{\phi}=10, k^{\theta}=5$, $k^{\psi}=10, k^{u}=5, k^{v}=10, k^{w}=1$, and $\varepsilon=$ 0.0001 . The initial position and orientation of the ROV are taken as: $[u, v, w, p, q, r, \phi, \theta, \psi, x, y, z]^{T}(0)$ $=[0.3,0,0,0,0,0,0.5,-0.1,0.2,0 ., 0,0.1]^{T}$. Figure 3 show the time evolution of the ROV state variables. Figures 5 and 6 depict the convergence of the roll, 


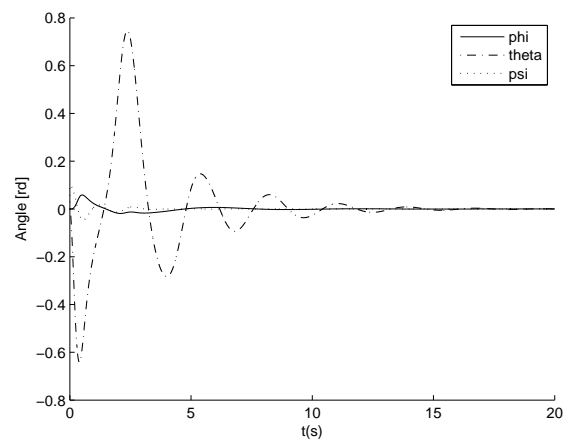

Figure 5: Behaviour of states $\phi, \theta$ and $\psi$

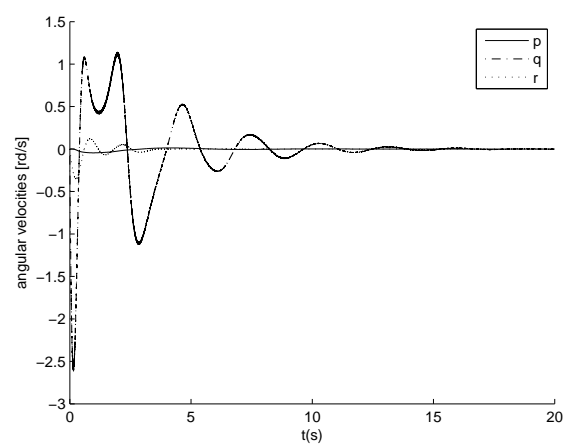

Figure 6: Behaviour of states $p, q$ and $r$

pitch, yaw and their derivatives, in a relatively short time. It is clear that the total ROV model (14) and (15) is exponentially stabilized at the origin using only three control inputs (17).

\section{CONCLUSIONS}

ROVs offer a control challenge as they have non zero drift. We proved that the ROV presented in the paper is not stabilizable by continuous pure state feedback law. The problem of asymptotic stabilization of the origin by means of smooth time-variant feedback law has been proposed for the kino-dynamic model. The averaging theory and the linearization technique are used to prove the stabilizing results. (Jiang, 2002)

\section{ACKNOWLEDGEMENTS}

This work is supported by the European Digital Ocean project under grant FP7 262160.

\section{REFERENCES}

Brockett, R. W. (1983). Asymptotic stability and feedback stabilization. In Differential geometric control theory, Progress in Math.

Chouiten, M., Domingues, C., Didier, J.-Y., Otmane, S., and Mallem, M. (2012). Distributed mixed reality for remote underwater telerobotics exploration. In Proceedings of the 2012 Virtual Reality International Conference, VRIC '12, pages 1:1-1:6, New York, NY, USA. ACM.

Coron, J.-M. (1992). Global asymptotic stabilisation for controllable systems without drift. In Math. of Control Signals and Systems.

Coron, J.-M. (1998). On the stabilization of some nonlinear control systems: results, tools, and applications. In NATO Advanced Study Institute, Montreal.

Coron, J.-M. and Rosier, L. (1994). A relation between continuous time-varying and discontiniues feedback stabilization. In J. Math. Systems Estimation and Control.

Cunha JP, Costa RR, H. L. (1995). Design of a high perfermance variable structure position control of rov's. In IEEE Journal of Oceanic Engineering.

Folcher, J. P. and Rendas, J. (2001). Identification and control of the phantom 500 body motion. In Proceedings of Oceans.

Fossen, T. I. (994). Guidence and Control of Ocean Vehicules. Chichester, Wiley.

Jiang, Z.-P. (2002). Brief global tracking control of underactuated ships by lyapunov's direct method. Automatica, 38(2):301-309.

Lapierre, L. and Soetanto, D. (2007). Nonlinear path following control of an auv. In Ocean Engineering.

Martins-Encarnacao, P. M. (2002). Nonlinear path following control system forocean vehicles. Universit Technique de Lisbonne. $\mathrm{PhD}$ thesis.

M'Closkey, R. T. and Murray, R. M. (1997). Exponential stabilization of driftless nonlinear control systems using homogeneous feedback. In IEEE Trans. Autom. Cont.

Morin, P. and Samson, C. (1997). Time-varying exponential stabilization of a rigid spacecraft with two control torques. In IEEE Trans. Autom. Cont.

Nakamura, M. Kjiwara, H. K. W. (2000). Development of an rov operated both as towed and self-propulsive vehicle. In Ocean Engineering 28.

Pettersen, K. Y. and Egeland, O. (1999). Time-varying exponential stabilization of the position and attitude of an underactuated autonomous underwater vehicle. In IEEE Trans. on Automatic Control.

Pettersen, K. Y. and Nijmeijer, H. (2001). Underactuated ship tracking control: theory and expriments. In Int. J. Control.

Santos, A. S. (1995). Contribution la conception des sousmarins autonomes. Ecole Nationale Suprieure des Mines de Paris. PhD thesis. 